\title{
Automating Strip Mapping
}

\author{
Aileen R. Buckley \\ a Esri, 380 New York Street, Redlands, CA, 92373m],USA, abuckley@esri.com
}

Keywords: Strip map, map automation, ArcGIS Pro

\begin{abstract}
:
Strip maps are used by cartographers to show the (primarily) spatial, (sometimes) attributional, and (rarely) temporal nature of linear geographic features. They have been used to show different types of linear features, such as the routes that travellers can follow (e.g., AAA TripTiks), the longest rivers of the world, and features with "some legal, administrative, or military significance" (MacEachren, 1986). Despite their unique format and myriad uses, these maps have often been overlooked as a form of cartographic representation (MacEachren, 1986). They have received little attention in cartographic literature, and they are not often found as cartographic products. When they are used appropriately and executed well, they are often lauded by cartographers; for example, Daniel Huffman's map of the coast of Lake Michigan, which enjoyed much popular acclaim (for example, Bliss, 2015); John Nelson's “Julie's Improbable Flight" which won the 2019 CaGIS Map Competition for Best Digital Map; and the scroll map of the Beijing-Hangzhou Grand Canal which won an award in the 2017 International Cartographic Exhibition.
\end{abstract}

More than 30 years ago, Dr. Alan MacEachren (1986) provided an excellent description of strip maps: "The following characteristics, with each successive characteristic generally incorporating the previous ones, representing increasing degrees of "stripness": a) linear form omitting geographic detail beyond a central corridor; b) orientation with a direction other than north at the top (with a non-cardinal direction being more indicative!; c) total lack of concern with geographic orientation, oriented in an unconventional direction and no indication of cardinal directions; d) relaxation of planimetric accuracy (changes in scale and orientation within map segments to adapt to the linear format); e) strict linear representation of a central feature with little consistency of either scale or direction".
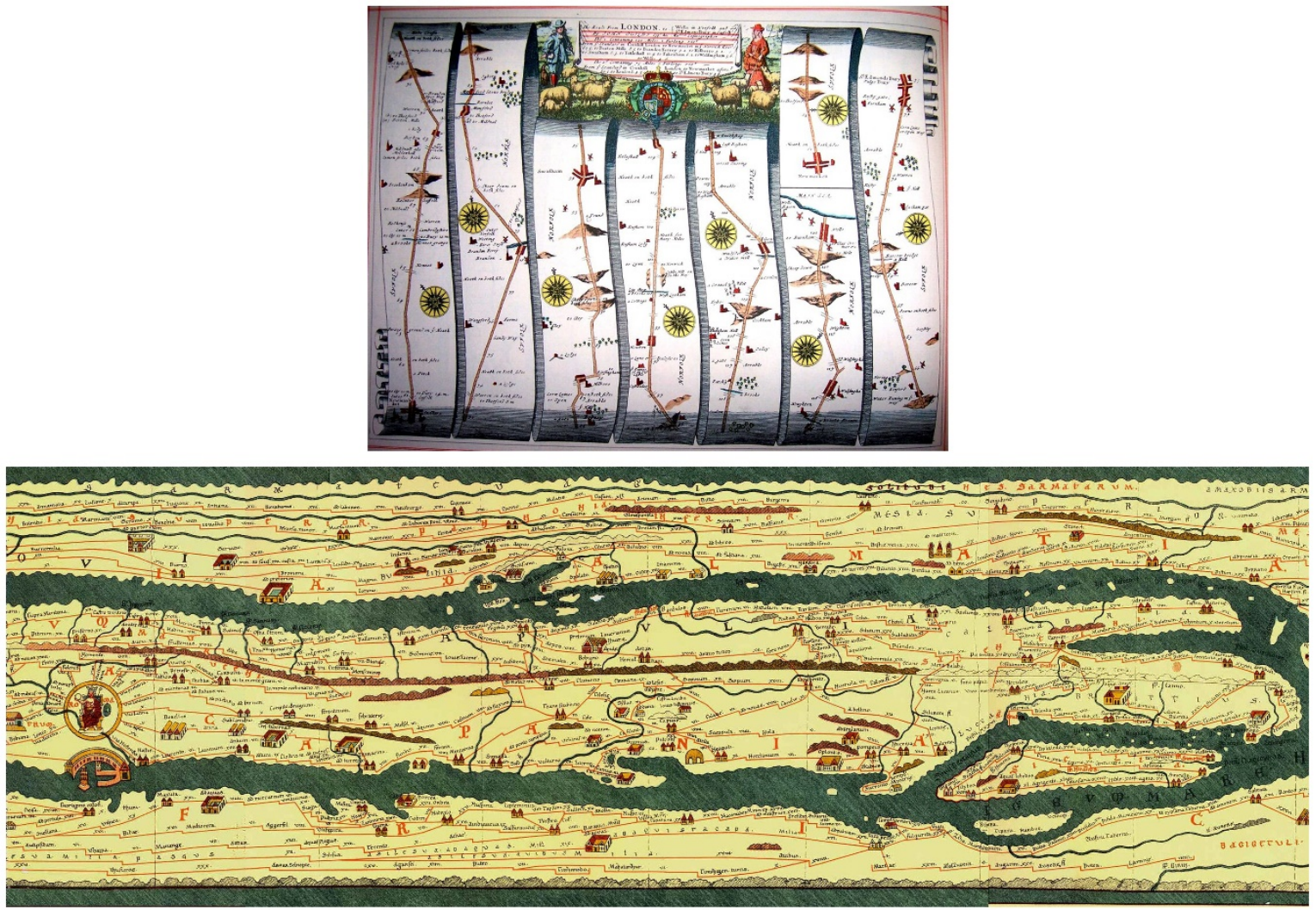

Figure 1. A portion of John Ogilby's strip map published in his 1675 Britannia Atlas (top); a portion of Peutiger's 22 foot long $13^{\text {th }}$ century Roman road map (bottom). 
Strip maps have a variety of uses, although the most prominent has been to show travel routes (MacEachren and Johnson, 1987). Strip maps that are linear route maps follow a route that is annotated with key landmarks, features adjacent to the route, and, often, the time it takes to travel between points along the route. An early example of this type of strip map is the massive work by John Ogilby published in his 1675 Britannia Atlas (Figure 1, top). This strip map is shown in segments that are read starting in the lower left, proceeding up to the top of the page, continuing down the next strip, then up the next, and so on. Another example is Peutiger's $13^{\text {th }}$ century illustrated ancient Roman road map that shows the road network of the Roman Empire printed on a continuous 22-foot long parchment scroll (Figure 1, bottom).

Another way to produce a strip map is as a set of map pages that follow a linear feature, such as a river, road, or pathway. Each page in the series shows a defined geographic area on either side of the linear feature. Each subsequent page in the strip map shows an area further down the line. Often, there is a bit of geographic overlap between adjacent map pages. The direction of north on the page shifts so that the flow of map is kept constant (Esri, 2021).

In ArcGIS Pro, strip maps can be created quickly and easily for multiple areas (for example, each of world's longest rivers). Using the geoprocessing tools in the Map Series toolset, data frame properties, and dynamic text, the process is largely automated. The line features used to determine the route of the strip map can be creating using editing tools, although an easier alternative is to use data from ArcGIS Living Atlas.

There are a number of important conditions that must be considered to create the most accurate and informative strip maps, including the page layout, map marginalia, map projection, and creation of the index, which is a series of rectangular polygons that follow a single linear feature or a group of linear features. The index features are used to define each page of the strip map by specifying the spatial extent and map rotation for each page, as well as providing data that can be used to label the previous and following pages.

In addition to mapping the linear features in a strip map fashion, ArcGIS also be used to automate the following: determining and labelling the predominant orientation of the linear feature on each page; determining and labelling distances along the linear features as either spatial distance (for example, miles or kilometers) or functional distance (for example, travel time); labelling heights along the linear features; showing characteristics of the landscape or geographic features within a narrow corridor along the linear feature, such as populated places, major roads, water bodies, political boundaries, terrain features, and more. Further, it is not difficult to image that attributes of the linear features could also be displayed. For example, a strip map of road features could show traffic counts, or a strip map of rivers could show streamflow values. Using GIS to automate strip mapping may result in increased popularity of these types of maps, and it may also contribute to more innovative uses for these types of maps.

\section{References:}

Bliss, Laura. 2015. The Coast of Lake Michigan Looks Extra Oceanic in this new "Linear Map". Bloomberg CityLab, https://www.bloomberg.com/news/articles/2015-10-19/daniel-huffman-s-linear-map-of-lake-michigan.

Esri. 2021. "Introduction to Spatial Map Series, https://pro.arcgis.com/en/pro-app/latest/help/layouts/spatial-mapseries.htm.

Jenny, Helen, Bernhard Jenny, William Cartwright \& Lorenz Hurni, Creating Panoramic Strip Format Maps, http://berniejenny.info/pdf/2011_Jenny_etal_Stripmaps.pdf.

Kimerling, A. Jon, Aileen R. Buckley, Phillip C. Muehrcke, \& Juliana O. Muehrcke. 2016. Map Use, Eight edition, California: Esri Press.

MacEachren, Alan M. \& Gregory B. Johnson. 1987. The Evolution, Application and Implications of Strip Format Travel Maps, The Cartographic Journal, 24:2, 147-158, DOI: 10.1179/caj.1987.24.2.147

MacEachren, Alan M. 1986. A Linear View of the World: Strip Maps as a Unique Form of Cartographic Representation, The American Cartographer, 13(1): 7-25 